ABHANDLUNGEN

DER AKADEMIE DER WISSENSCHAFTEN DER DDR

Abteilung Veröffentlichung der Wissenschaftlichen Räte

Jahrgang 1986

Gemeinsame Tagung des Wissenschaftlichen Rates

für die wirtschaftswissenschaftliche Forschung bei der Akademie der Wissenschaften der DDR und seines Wissenschaftlichen Rates für Fragen des sozialistischen Wettbewerbs vom 30.5.1985

\title{
Sozialistischer Wettbewerb
}

und

umfassende Intensivierung 
Herausgegeben im Auftrage des Präsidenten der Akademie der Wissenschaften der DDR von Vizepräsident Prof. Dr. Heinz Stiller

Verantwortlich für dieses Heft:

Akademiemitglied Prof. Dr.sc. oec. Dr.h.c. Helmut Koziolek Vorsitzender des Wissenschaftlichen Rates

für die wirtschaftswissenschaftliche Forschung

bei der Akademie der Wissenschaften der DDR

Redaktionsschluß: 15. September 1985

ISSN: 0138-421 X

Erschienen im Akademi_-Verlag Berlin, DDR - 1086 Berlin,

Leipziger Str. 3-4

(c) Akademie-Verlag Berlin 1985

Lizenznummer: $202 \cdot 100 / 225 / 86$

Printed in the German Democratic Republic

Gesamtherstellung: TASTOMAT, 1275 Eggersdorf,

Landhausstraße

LSV 0345

Bestellnummer: $7546801(2001 / 86 / 1 / w)$

01400 


\section{THESEN}

\section{REFERAT}

Prof. Dr. Horst Schneider

Mitglied des Wissenschaftlichen Rates für die wirtschaftswissenschaftliche Forschung bei der Akademie der Wissenschaften der DDR,

Vorsitzender des Wissenschaftlichen Rates für Fragen des sozialistischen Wettbewerbs,

Direktor der Gewerkschaftshochschule „Fritz Heckert" beim Bundesvorstand des FDGB

Sozialistischer Wettbewerb und umfassende Intensivie-

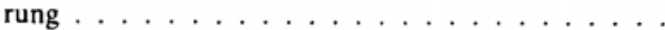

\section{DISKUSSION}

Horst Heintze

Mitglied des $Z K$ der SED,

Mitglied des Präsidiums und Sekretär des Bundesvor. standes des FDGB

Zur Entwicklung des sozialistischen Wettbewerbs in Vorbereitung des XI. Parteitages der SED . . . . . .

Fritz Brock

Mitglied der Zentralen Revisionskommission des $Z K$ der $S E D$, Leiter der Abteilung Gewerkschaften und Sozialpolitik des $Z K$ der SED

Konsequent den sozialistischen Wettbewerb auf die umfassende Intensivierung einstellen . . . . . . . . . .

Dr.sc. Erhard Dörschel

Akademie für Gesellschaftswissenschaften beim $Z K$ der $S E D$, Institut für Politische Ökonomie des Sozialismus Sozialistischer Wettbewerb und Vervollkommnung der sozialistischen Produktionsverhältnisse . . . . . . . .

Prof. Dr. Waltraud Falk

Dekan der gesellschaftswissenschaftlichen Fakultät der

Humboldt-Universität zu Berlin

und Prof. Dr. Horst Barthel

Humboldt-Universität zu Berlin, Sektion Wirtschaftswissenschaften

Zur Geschichte der organisierten sozialistischen Masseninitiative und aktuelle Lehren ..........

Prof. Dr. Willi Kunz

Korrespondierendes Mitglied der Akademie der Wissenschaften der DDR, Mitglied des Wissenschaftlichen Rates für die wirtschaftswissenschaftliche Forschung bei der Akademie der Wissenschaften der DDR,

Vorsitzender des Wissenschaftlichen Rates für Fragen der sozialistischen ökonomischen Integration.

Stellvertreter des Direktors des Zentralinstituts für sozialistische Wirtschaftsführung beim $Z K$ der SED

Erfahrungsaustausche und Leistungsvergleiche zwischen Kombinaten und Betrieben zur Erschließung von Leistungsreserven* . . . . . . . . . . . . . . .
Seite

5 Prof. Dr. Karl Hartmann

Stellvertretender Vorsitzender des Wissenschaftlichen Rates für die wirtschaftswissenschaftliche Forschung bei der Akademie der Wissenschaften der DDR,

Direktor des Wissenschaftsbereichs Politische Ökonomie und Wirtschaftswissenschaften der Parteihochschule „Karl Marx" beim ZK der SED

Wissenschaftlich-technischer Fortschritt und sozialistischer Wettbewerb* . . . . . . . . . . . . . . .

Peter Siewert

Vertrauensmann des Kollektivs der Techniker des VEB Automobilwerke Ludwigsfelde

$\mathrm{Zu}$ Ansprüchen an die Wettbewerbsführung im Bereich der Technologie im Stammbetrieb des IFA-Kombinates Nutzkraftwagen bei der Beschleunigung der wissenschaftlich-technischen Entwicklung .........

Jürgen Radke

Sekretär für Arbeit und Löhne des Kreisvorstandes der IG Chemie, Glas, Keramik

Forscherkonten - eine im Leuna-Kombinat entwickelte und bewährte Wettbewerbsinitiative

Dr.sc. Klaus Gebauer

Zentralinstitut für sozialistische Wirtschaftsführung beim $Z K \operatorname{der} S E D$

Sozialistischer Wettbewerb und Stimulierung in Forschung und Entwicklung der Kombinate . . . . . . .

Prof. Dr. Joachim Neumann

Technische Universität Dresden, Sektion Sozialistische Betriebswirtschaft

und Dr. Gerhard Jesse

Technische Universität Dresden, Sektion Arbeitswissenschaften

Zur Organisation der Neuerertätigkeit als Bestandteil der Erneuerung der Produktion in den Kombinaten und Betrieben .................

Prof. Dr. Ursula Gabler

Mitglied des Wissenschaftlichen Rates für die wirtschafiswissenschaftliche Forschung bei der Akademie der Wissenschaften der DDR,

Friedrich-Schiller-Universität Jena, Sektion Wirtschaftswissenschaften

Nutzung von Planungsmethoden für die Wettbewerbs-

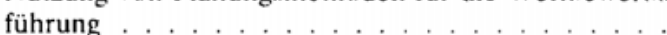

Werner Wollschlaeger

40 Sekretär für Arbeit und Löhne des Bezirksv'orstundes de's FDGB Berlin

Erfahrungen in der Leitungstätigkeit bei der Entwicklung der schöpferischen Masseninitiative zur Verwirklichung der ökonomischen Strategie. insbesondere zur Beschleunigung des wissenschaftlich-technischen Fortschritts*

Prof. Dr. Rolf Rinke

Vorsitzender des Zentralvorstandes der Gewerkschaft Wissenschaft

Erfahrungen und Ergebnisse bei der Erhöhung der Wirksamkeit des sozialistischen Wettbewerbs in Akade- 
Heinrich Brodowski

Sekretär der Parteiorganisation der SED des VEB Reifenkombinats Fürstenwalde

Neue Aspekte der Arbeit mit Notizen zum Plan im sozialistischen Wettbewerb aus der Sicht der Leitungstätigkeit im Stammbetrieb des VEB Reifenkombinat Für-

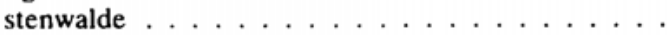

Dr. Dieter Weger

Sekretär der Bezirksleitung der SED Frankfurt (Oder)

Zur politischen Führung der schöpferischen Masseninitiative im sozialistischen Wettbewerb durch die Bezirksleitung der SED Frankfurt (Oder) . . . . . . .

\section{Jürgen Hunneshagen}

BGL-Vorsitzender des VEB Werkzeugmaschinenkombinats „7. Oktober" Berlin-Weißensee

Erfahrungen bei der Führung des sozialistischen Wettbewerbs in den integrierten Fertigungsabschnitten des Stammbetriebes des VEB Werkzeugmaschinenkombinats „7. Oktober" Berlin-Weißensee * . . . . . . . . .

Gerhard Patsch

BGL-Vorsitzender des VEB PCK Schwed

Die neuen Anforderungen an die Verwirklichung der

Schwedter Initiative ${ }^{*} \ldots \ldots . . . .$.

Prof. Dr. Gunnar Winkler

Mitglied des Wissenschaftlichen Rates für die wirtschaftswissenschaftliche Forschung bei der Akademie der Wissenschaften der DDR,

Vorsitzender des Wissenschaftlichen Rates für Fragen der Sozialpolitik und Demografie,

Direktor des Instituts für Soziologie und Sozialpolitik der Akademie der Wissenschaften der DDR

Soziale Aspekte des sozialistischen Wettbewerbs . .

\section{Dr. Karin Schießl}

Gewerkschaftshochschule „Fritz Heckert" beim Bundesvorstand des FDGB, Leiter des Lehrstuhls marxistischleninisiische Philosophie
Gemeinschaftsarbeit und Gewerkschaftsarbeit im

Neuerungsproze $B \ldots \ldots \ldots \ldots$

Prof. Dr. Jürgen Wahse

Akademie der Wissenschaften der DDR, Zentralinstitut für Wirtschaftswissenschaften

69 Zu einigen aktuellen Problemen der effektiven Nutzung des gesellschaftlichen Arbeitsvermögens* . . . . . .

Prof. Dr.-Ing. Wilma Podewin

Mitglied des Wissenschaftlichen Rates für die wirtschaftswissenschaftliche Forschung bei der Akademie der Wissenschaften der DDR,

Humboldt-Universität zu Berlin, Sektion Wissenschaftstheorie und Organisation

Den sozialistischen Wettbewerb und die wissenschaftliche Arbeitsorganisation eng miteinander verbinden*

Dietmar Ketzel

Stellvertreter des Direktors des VEG Pflanzenproduktion Schwaneberg

Sozialistischer Wettbewerb im VEG Pflanzenproduktion Schwaneberg und Anforderungen an die sozialistische Arbeitsorganisation . . . . . . . . . . .

\section{SCHLUSSWORT}

Prof. Dr. Dr.h.c. Helmut Koziolek Mitglied des $Z K$ der SED, Ordentliches Mitglied der Akademie der Wissenschaften der DDR, Vorsitzender des Wissenschaftlichen Rates für die wirtschaftswissenschaftliche Forschung bei der Akademie der Wissenschaften der DDR,

Direktor des Zentralinstituts für sozialistische Wirtschaftsführung beim $Z K$ der SED

* schriftlich eingereichte Beiträge 